\title{
Lower Bounds of the Kirchhoff and Degree Kirchhoff Indices
}

\author{
I. Ž. Milovanović, E. I. Milovanović, E. Glogić
}

Paper dedicated to memory of Vera Nikolić-Stanojević, Pofessor Emeritus at the State University of Novi Pazar

\begin{abstract}
Let $G$ be an undirected connected graph with $n, n \geq 3$, vertices and $m$ edges. If $\mu_{1} \geq \mu_{2} \geq \cdots \geq \mu_{n-1}>\mu_{n}=0$ and $\rho_{1} \geq \rho_{2} \geq \cdots \geq \rho_{n-1}>\rho_{n}=0$ are the Laplacian and the normalized Laplacian eigenvalues of $G$, then the Kirchhoff and the degree Kirchhoff indices obey the relations $K f(G)=n \sum_{i=1}^{n-1} \mu_{i}^{-1}$ and $D K f(G)=2 m \sum_{i=1}^{n-1} \rho_{i}^{-1}$, respectively. The inequalities that determine lower bounds for some invariants of $\mathrm{G}$, that contain $K f(G)$ and $D K f(G)$, are obtained in this paper. Lower bounds for $K f(G)$ and $D K f(G)$, known in the literature, are obtained as a special case.
\end{abstract}

Keywords: Kirchhoff index, Degree Kirchhoff index, Laplacian spectrum (of graph), normalized Laplacian spectrum (of graph).

\section{Introduction}

Let $G$ be undirected connected graph with $n, n \geq 3$, vertices and $m$ edges, with vertex degree sequence $\Delta=d_{1} \geq d_{2} \geq \cdots \geq d_{n}=\delta>0$. Denote by $A$ the adjacency matrix of the graph $G$ and by $D$ the diagonal matrix of its vertex degrees. Then $L=D-A$ is Laplacian matrix of $G$. Denote by $\mu_{1} \geq \mu_{2} \geq \cdots \geq \mu_{n-1}>\mu_{n}=0$ eigenvalues of $L$ (see $[8,18]$ ). Because the graph $G$ is assumed to be connected it has no isolated vertices and therefore the matrix $D^{-1 / 2}$ is well defined. The $L^{*}=D^{-1 / 2} L D^{-1 / 2}$ is normalized Laplacian matrix of the graph $G$. Its eigenvalues are $\rho_{1} \geq \rho_{2} \geq \cdots \geq \rho_{n-1}>\rho_{n}=0$. For details of spectral theory of the normalized Laplacian matrix see, for example, [6].

For Laplacian and normalized Laplacian eigenvalues the following equalities are valid

$$
\sum_{i=1}^{n-1} \mu_{i}=\sum_{i=1}^{n} d_{i}=2 m, \quad \sum_{i=1}^{n-1} \mu_{i}^{2}=\sum_{i=1}^{n} d_{i}^{2}+\sum_{i=1}^{n} d_{i}=M_{1}+2 m
$$

Manuscript received May 21, 2014; accepted October 4, 2014.

I. Ž. Milovanović, E. I. Milovanović are with the Faculty of Electrical Engineering, Niš, Serbia; E. Glogić is with the State University of Novi Pazar, Novi Pazar, Serbia; 
and

$$
\sum_{i=1}^{n-1} \rho_{i}=n, \quad \sum_{i=1}^{n-1} \rho_{i}^{2}=n+2 R_{-1},
$$

where $M_{1}$ is the first Zagreb index (see [14]), and $R_{-1}$ is Randić index (see [4, 24]).

Graph invariant named Kirchhoff index, is defined as [13]

$$
K f(G)=n \sum_{i=1}^{n-1} \frac{1}{\mu_{i}}
$$

and the degree Kirchhoff index as [5]

$$
D K f(G)=2 m \sum_{i=1}^{n-1} \frac{1}{\rho_{i}}
$$

The graph invariants $K f$ and $D K f$ are currently much studied in the mathematical and mathematico chemical literature; see the recent papers $[1,9,10,11,12,15,16,20,21]$ and the references cited therein. In a few cases these invariants can be determined in a closed form. Therefore, the inequalities that give upper or lower bounds of these invariants are notable. The bounds can be determined in terms of usual structural parameters, such as number of vertices, number of edges, vertex degrees, and similar, or extremal Laplacian and normalized Laplacian eigenvalues, or Zagreb or Randić index, etc. (see [2, 3, 9, 10, 15, $16,17,20,22,23]$. In this paper we consider lower bounds for some graph invariants that, in a special case, reduce to $K f(G)$ and $D K f(G)$.

\section{Main result}

We first give two inequalities for non-negative real numbers.

Theorem 1 Let $a_{1}, a_{2}, \ldots, a_{n}$ be non-negative real numbers with the property $a_{1}+a_{2}+\cdots+$ $a_{n}=A>0$. In addition, let $l, r>0$ and $p \geq 1$ be numbers with the property $r(j-p+1)+l \geq$ 1 or $r(j-p+1)+l<0$, for each $j, j \geq p-1$. Then the following is valid

$$
\sum_{i=1}^{n} \frac{a_{i}^{l}}{\left(A^{r}-a_{i}^{r}\right)^{p}} \geq \frac{n^{p r-l+1} A^{l-p r}}{\left(n^{r}-1\right)^{p}}
$$

Equality holds if and only if $a_{1}=a_{2}=\cdots=a_{n}$.

Proof Suppose that numbers $l, r$ and $p$ satisfy the conditions of Theorem 1 . Then, for each $a_{i}, 0 \leq a_{i} \leq A, i=1,2, \ldots, n$ the following inequality is valid

$$
\frac{a_{i}^{l}}{\left(A^{r}-a_{i}^{r}\right)^{p}}=\frac{1}{(p-1) !} \sum_{j=p-1}^{+\infty} \frac{(j)_{p-1}}{A^{r(j+1)}} a_{i}^{r(j-p+1)+l},
$$


where $(j)_{p-1}=j(j-1) \cdots(j-p+2),(j)_{0}=j$. Summing the above inequality on $i$, $i=1,2, \ldots, n$, we obtain

$$
\begin{aligned}
\sum_{i=1}^{n} \frac{a_{i}^{l}}{\left(A^{r}-a_{i}^{r}\right)^{p}} & =\sum_{i=1}^{n} \frac{1}{(p-1) !} \sum_{j=p-1}^{+\infty} \frac{(j)_{p-1}}{A^{r(j+1)}} a_{i}^{r(j-p+1)+l}= \\
& =\frac{1}{(p-1) !} \sum_{j=p-1}^{+\infty} \frac{(j)_{p-1}}{A^{r(j+1)}} \sum_{i=1}^{n} a_{i}^{r(j-p+1)+l}
\end{aligned}
$$

When apply discrete Jensen inequality (see [19]) to the above inequality we obtain

$$
\begin{aligned}
\sum_{i=1}^{n} \frac{a_{i}^{l}}{\left(A^{r}-a_{i}^{r}\right)^{p}} & \geq \frac{n}{(p-1) !} \sum_{j=p-1}^{+\infty} \frac{(j)_{p-1}}{A^{r(j+1)}}\left(\frac{\sum_{i=1}^{n} a_{i}}{n}\right)^{r(j-p+1)+l}= \\
& =\frac{n\left(\frac{A}{n}\right)^{l}}{\left(A^{r}-A^{r} n^{-r}\right)^{p}}=\frac{n^{p r-l+1} A^{l-p r}}{\left(n^{r}-1\right)^{p}}
\end{aligned}
$$

The following theorem can be similarly proved.

Theorem 2 Suppose that real numbers $a_{1}, a_{2}, \ldots, a_{n}$ and numbers $l, r$ and $p$ satisfy the conditions of Theorem 1. Then

$$
\sum_{i=1}^{n} \frac{a_{i}^{l}}{\left(A^{r}-a^{r}\right)^{p}} \geq \frac{a_{1}^{l}}{\left(A^{r}-a_{1}^{r}\right)^{p}}+\frac{(n-1)^{p r-l+1}\left(A-a_{1}\right)^{l-p r}}{\left((n-1)^{r}-1\right)^{p}} .
$$

Equality holds if and only if $a_{2}=a_{3}=\cdots=a_{n}$.

We now obtain an lower bounds for some graph invariants.

Theorem 3 Let $G$ be an undirected connected graph with $n, n \geq 3$, vertices and $m$ edges. Iffor numbers $l$ and $p, p \geq 1$, hold $j-p+l \geq 0$ for each $j, j \geq p-1$, then

$$
\sum_{i=1}^{n-1} \frac{\left(2 m-(n-2) \mu_{i}\right)^{l}}{\mu_{i}^{p}} \geq(n-1)^{p-l+1}(2 m)^{l-p}
$$

Equality holds if and only if $G \cong K_{n}$.

Proof. For $n:=n-1, r=1, a_{i}=A-\mu_{i}, i=1,2, \ldots, n-1$, inequality (3) becomes

$$
\sum_{i=1}^{n-1} \frac{\left(A-\mu_{i}\right)^{l}}{\mu_{i}^{p}} \geq \frac{(n-1)^{p-l+1} A^{l-p}}{(n-2)^{p}}
$$


Based on the equality $a_{i}=A-\mu_{i}, i=1,2, \ldots, n-1$ and (1), we obtain that $A=\frac{2 m}{n-2}$. By substituting $A=\frac{2 m}{n-2}$ in (6) we arrive at (5). Having in mind that equality in (3), for $n:=n-1$, occurs if and only if $a_{1}=a_{2}=\cdots=a_{n-1}$, equality in (5) holds if and only if $G \cong K_{n}$.

Corollary 1 Let $G$ be undirected connected graph with $n, n \geq 3$, vertices and $m$ edges. Then, for each $p, p \geq 1$ the following is valid

$$
\sum_{i=1}^{n-1} \frac{1}{\mu_{i}^{p}} \geq \frac{(n-1)^{p+1}}{(2 m)^{p}}
$$

Equality holds if and only if $G \cong K_{n}$.

Corollary 2 Let $G$ be undirected connected graph with $n, n \geq 3$, vertices and $m$ edges. Then

$$
K f(G) \geq \frac{n(n-1)^{2}}{2 m} \geq \frac{(n-1)^{2}}{\Delta}
$$

Equality holds if and only if $G \cong K_{n}$.

Theorem 4 Let $G$ be undirected connected graph with $n, n \geq 3$, vertices and $m$ edges. Then, for each $p, p \geq 1$

$$
\sum_{i=1}^{n-1} \frac{1}{\left(1-\frac{\mu_{i}}{2 m}\right)^{p}} \geq \frac{(n-1)^{p+1}}{(n-2)^{p}} .
$$

Equality holds if and only if $G \cong K_{n}$.

Corollary 3 Let $G$ be undirected connected graph with $n, n \geq 3$, vertices and $m$ edges. Then, for each $p, p \geq 1$

$$
\sum_{i=1}^{n-1} \frac{1}{\mu_{i}^{p}} \geq \frac{1}{n^{p}}+\frac{(n-2)^{p+1}}{(2 m-\Delta-1)^{p}} .
$$

Equality holds if and only if $G \cong K_{n}$.

Corollary 4 Let $G$ be undirected connected graph with $n, n \geq 3$, vertices and $m$ edges. Then

$$
K f(G) \geq 1+\frac{n(n-2)^{2}}{2 m-\Delta-1} .
$$

Equality holds if and only if $G \cong K_{n}$. 
Corollary 5 [22] Let $G$ be undirected connected graph with $n, n \geq 3$, vertices and $m$ edges. Then

$$
\sum_{i=1}^{n-1} \frac{n}{\mu_{i}^{p}} \geq \frac{n}{(1+\Delta)^{p}}+\frac{n(n-2)^{p+1}}{(2 m-\Delta-1)^{p}} .
$$

Equality holds if and only if $G \cong K_{n}$.

Corollary 6 [23] Let $G$ be undirected connected graph with $n, n \geq 3$, vertices and $m$ edges. Then

$$
K f(G) \geq \frac{n}{1+\Delta}+\frac{n(n-2)^{2}}{2 m-\Delta-1} .
$$

Equality holds if and only if $G \cong K_{n}$.

Corollary 7 Let $G$ be undirected connected graph with $n, n \geq 3$, vertices and $m$ edges. Then

$$
K f(G) \geq \frac{n^{2}(n-1)-2 m}{2 m} \geq n-1 .
$$

Equality holds if and only if $G \cong K_{n}$.

We now obtain inequalities that in a special case assign lower bound for graph invariant $D K f(G)$.

Theorem 5 Let $G$ be undirected connected graph with $n, n \geq 3$, vertices and $m$ edges. Then, for each $l$ and $p, p \geq 1$, that obey inequality $j-p+l \geq 0$, for each $j, j \geq p-1$, the following is valid

$$
\sum_{i=1}^{n-1} \frac{\left(n-(n-2) \rho_{i}\right)^{l}}{\rho_{i}^{p}} \geq(n-1)^{l-p+1} n^{l-p} .
$$

Equality holds if and only if $G \cong K_{n}$.

Proof. For $n:=n-1, r=1, a_{i}=A-\rho_{i}, i=1,2, \ldots, n-1$, inequality (3) transforms into

$$
\sum_{i=1}^{n-1} \frac{\left(A-\rho_{i}\right)^{l}}{\rho_{i}^{p}} \geq \frac{(n-1)^{p-l+1} A^{l-p}}{(n-2)^{p}} .
$$

According to the equality $a_{i}=A-\rho_{i}$ and (2) we have that $A=\frac{n}{n-2}$. Substituting $A=\frac{n}{n-2}$ in the above inequality we arrive at (7).

For $n:=n-1$, equality in (3) holds if and only if $a_{1}=a_{2}=\cdots=a_{n-1}$, so the equality in (7) holds if and only if $\rho_{1}=\rho_{2}=\cdots=\rho_{n-1}$, i.e. when $G \cong K_{n}$. 
Corollary 8 Let $G$ be undirected connected graph with $n, n \geq 3$, vertices and $m$ edges. Then, for each $p, p \geq 1$

$$
\sum_{i=1}^{n} \frac{2 m}{\rho_{i}^{p}} \geq \frac{2 m(n-1)^{p+1}}{n^{p}}
$$

Equality holds if and only if $G \cong K_{n}$.

Corollary 9 [21] Let $G$ be undirected connected graph with $n, n \geq 3$, vertices and $m$ edges. Then

$$
D K f(G) \geq \frac{2 m(n-1)^{2}}{n} .
$$

Equality holds if and only if $G \cong K_{n}$.

Theorem 6 Let $G$ be undirected connected graph with $n, n \geq 3$, vertices and $m$ edges. Then, for each $p, p \geq 1$

$$
\sum_{i=1}^{n-1} \frac{2 m}{\left(n-\rho_{i}\right)^{p}} \geq \frac{2 m(n-1)^{p+1}}{n^{p}(n-2)^{p}}
$$

Equality holds if and only if $G \cong K_{n}$.

\section{Acknowledgement}

This research was supported by the Serbian Ministry of Education and Science, under grant TR32012.

\section{References}

[1] C. Arauz, The Kirchhoff indexes of some composite networks, Discrete Appl. Math., 160 (2012), 1429-1440.

[2] M. Biachi, A. Cornaro, J. L. Palacis, A. Torriero, Bound for Kirchhoff index via majorizatio techniques, J. Math. Chem., 51 (2013), 569-587.

[3] S. B. BOZKURT, D. BOzKURT, On the sum of powers of normalized Laplacian eigenvalues of graph, MATCH Commun. Math. Comput. Chem., 68 (2012), 917-930.

[4] M. CAVERS, S. FAllat, S. Kirkland, On the normalized Laplacian energy and the general Randić index $R_{-1}$ of graphs, Lin. Algebra Appl., 433 (2010), 172-190.

[5] H. Chen, F. Zhang, Resistance distance and the normalized Laplacian spectrum, Dis. Appl. Math., 155 (2007), 654-661.

[6] F. R. K. Chung, Spectral graph theory, Am. Math. Soc., Providence, 1997.

[7] S. M. Сіодв Â, Sums of powers of the degrees of a graph, Discrete Math., 306 (16) (2006), 1959-1964. 
[8] D. Cvetković, P. Rowlinson, S. K. Simić, Introduction to the theory of graph spectra, Cambridge Univ. Press, Cambridge, 2010.

[9] K.C. DAS, A. D. GUnGÖR, A. S. CEVIK, On Kirchhoff index and resistance distance energy of a graph, MATCH Commun. Math. Comput. Chem., 67 (2012), 541-556.

[10] L. Feng, I. Gutman, G. Yu, Degree Kirchhoff index of unicuclic graphs, MATCH Commun. Math. Comput. Chem., 69 (2013), 629-648.

[11] X. Gao, Y. LuO, W. LiU, Resistance distances and the Kirchhoff index in Cayley graphs, Discrete. Appl. Math., 159 (2011), 20150-2057.

[12] X. Gao, Y. Luo, W. LiU, Kirchhoff index in line subdivision of total graphs of a regular graph, Discrete. Appl. Math., 160 (2012), 560-565.

[13] I. Gutman, B. Mohar, The quasi-Wiener and the Kirchhoff indices coincite, J. Chem. Inf. Comput. Sci., 36 (1996), 982-985.

[14] I. Gutman, N. TRINAJEsić, Graph theory and molecular orbitals. Total $\pi$-electron energy of alternant hydrocarbons, Chem. Phys. Lett., 17 (1972), 535-538.

[15] M. Hakimi-NezhaAd, A. R. Ashrafi, I. Gutman, Note on degree Kirchhof index of graphs, Trans. Comb. 2 (3) (2013), 43-52.

[16] R. LI, Lower bounds for the Kirchhoff index, MATCH Commun. Math. Comput. Chem.,70 (2013), 163-174.

[17] M. LiU, B. LiU, A note on sums of powers of the Laplacian eigenvalues, Appl. Math. Letters, 24 (2011), 249-252.

[18] R. MERRIS, Laplacian matrices of graphs: a survey, Lin. Algebra Appl., 197-198 (1994), 143-175.

[19] D. S. Mitrinović, P. M. Vasić, Analytic inequalities, Springer, Berlin, 1970.

[20] J. L. Palacios, J. M. Renom, Another look at the degree Kirchhoff index, Int. J. Quantum Chem., 111 (2011), 3453-4355.

[21] J. PAlacios, J. M. RENOM, Brodr and Karlin's formule for hitting times and the Kirchhoff index, Int. J. Quantum Chem., 111 (2011), 35-39.

[22] B. ZHOU, On sum of powers of the Laplacian eigenvalues of graphs, Lin. Algebra Appl.,429 (2008), 2239-2246.

[23] B. Zhou, N. Trinajestić, A note on Kirchhoff index, Chem. Phys. Lett. 445 (2008), 120123.

[24] G. YU, L. FEng, Randić index and eigenvalues of graphs, Rocky Mountain J. Math., 40 (2010), 713-721. 